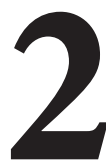

\title{
INVESTIGADORAS CON ÉXITO EN LA UNIVERSIDAD... ¿CÓMO LO HAN LOGRADO?
}

\section{(SUCCESSFUL WOMEN RESEARCHERS AT THE UNIVERSITY... HOW HAVE THEY COME TO ACHIEVE IT?)}

Mar Duran-Bellonch

Universidad Autónoma de Barcelona

Georgeta Ion

Universidad Autónoma de Barcelona

DOI: 10.5944/educxx1.17.1.10704

\section{Cómo referenciar este artículo/How to reference this article:}

Durán-Bellonch, M. e Ion, G. (2014). Investigadoras con éxito en la universidad... ¿Cómo lo han logrado? Educación XX1, 17 (1), 39-58. doi: 10.5944/educxx1.17.1.10704.

Durán-Bellonch, M. e Ion, G. (2014). Successful women researchers at the university... How have they done? Educación XX1, 17 (1), 39-58. doi:10.5944 /educxx1.17.1.10704.

\section{RESUMEN}

Presentamos los resultados de una investigación en la que nos preguntamos por las condiciones que influyen en el éxito de la actividad investigadora de las mujeres académicas en ciencias sociales. Consideramos que las mismas investigadoras pueden ofrecernos información valiosa al respecto, y por ello entrevistamos en profundidad a una muestra de académicas de una universidad pública catalana, todas ellas líderes de grupos de investigación reconocidos por la Generalitat de Catalunya en la actualidad. Partimos de un guión de entrevista compuesto por tres categorías teóricas: condicionantes para el éxito académico de carácter individual, condicionantes de tipo grupal y condicionantes institucionales. Los resultados validan las categorías teóricas y contribuyen a su enriquecimiento, en tanto que apuntan la existencia de una serie de subcategorías que pueden ser estudiadas en profundidad en futuras investigaciones. Destacan los siguientes factores individuales para el éxito en la carrera académica: "el amor por el saber» de quien investiga; su procedencia sociocultural; las estancias de investigación en el extranjero; la perseverancia y tenacidad; el apoyo de la familia; el respeto a los tiempos biológicos y la relación con un mentor o mentora. De entre los factores grupales aparecen: el equilibrio entre trabajo individual y grupal; la formación fruto del intercambio entre miembros del equipo de trabajo; la participación en equipo en proyectos competitivos y un estilo de liderazgo basado en la mediación y la delegación. Finalmente, de factores institucionales surgen con fuerza: la existencia de otros grupos de investigación con los que com- 
petir; la convergencia temática entre investigación y docencia; la dirección de trabajos de investigación y el soporte económico de órganos estatales y europeos.

\section{ABSTRACT}

We present the results of a research analysing the conditions that influence the successful research career of academic women in the field of the social sciences. In our research we used in-depth interview in a sample of academics pertaining to Catalan public universities, all of them leaders of research groups recognized by the Generalitat de Catalunya. The interviews were structured according to the following topics: individual conditions for academic success, group-type conditions and institutional factors.

The results validated the theoretical categories and contributed to their enrichment, while - nevertheless - pointing the existence of a number of subcategories that can be studied in depth in future research. Among the individual factors for success in academic careers participants mentioned: «the passion for knowing», the sociocultural factors, the research periods abroad, the perseverance and tenacity, the support of family, respect of biological time and the relationship with a mentor.

Among the factors linked to the group, we can highlight: the balance between individual and group work, the research experience exchange between team members, participation in competitive team projects and a leadership style based on mediation and delegation. Finally, the following institutional factors were also highlighted: the existence of other research groups with which they compete, the convergence between research and teaching activities, the coordination of research project and financial support from national and European bodies.

\section{INTRODUCCIÓN}

En las últimas décadas abundan los estudios dedicados al papel de la mujer en la universidad. Flecha (1999) expone los logros de las mujeres en Europa y España y los retos que entrando en el nuevo milenio todavía estaban por conseguir. Según la autora, los llamados «estudios de la mujer» contribuyen a repensar modelos que perpetúan la desigualdad de género en la educación superior. Aún con todo, «las mujeres no están representadas de manera igualitaria respecto a los hombres a lo largo de las categorías profesionales del sistema científico» (Bermúdez, y otros, 2011, p. 17). Es la llamada segregación vertical. Los resultados obtenidos en el reciente estudio experimental de Milkman, Akinola y Chugh (2012) son sugerentes en tanto que descubren que una de las razones para dicha segregación es que los académicos tienen acceso a información que les permite planificar su carrera 
a largo plazo, mientras que las académicas y las personas pertenecientes a minorías étnicas, no tienen las mismas facilidades de acceso, lo que solamente les permite tomar decisiones sobre su carrera a corto y medio plazo.

Además, a pesar de que el número de académicas se ha venido incrementando con los años, la academia sigue considerándose elitista, masculina y patriarcal (Asmar, 1999; Pole Bornholt, y Summers, 1997; Marie y Moldovan, 2012; Seierstad, 2012; Brink y Benschop, 2012, entre otros), puesto que la asignación de funciones sigue perpetuando el modelo tradicional (Sagaria y Agans, 2006; Smeby y Try, 2005). Un ejemplo en este sentido es que las mujeres tienen más carga docente que los hombres y reciben encargos de gestión que conllevan mucho trabajo y poco poder (McLaughlin y Helsi, 2013). Ellos, en cambio, se dedican y destacan más por su actividad investigadora (Bagilhole y White, 2003) y de gestión asociada a puestos con poder (Bagilhole, 2007; Ruth, 2005; Tomàs, Duran, Guillamon y Lavié, 2008).

Guillamon (2011) estudia las condiciones que llevan a que las mujeres tengan menos productividad investigadora que los hombres. Como obstáculos indica que las mujeres tienen menos confianza en sus habilidades y menos acceso a las redes académicas (Britton, 1999; Doherty y Manfredi, 2005) y optan en mayor medida por las denominadas «ciencias blandas», como son las humanidades y las ciencias sociales. Mientras tanto, los hombres tienen más presencia en las áreas denominadas «duras» como las ciencias empíricas o las tecnológicas.

Mientras la investigación representa solo una de las facetas del trabajo académico (junto a la docencia y a las tareas de gestión), Hobson, Jones y Deane (2005) observan como en la universidad actual, la investigación se considera como el aspecto más importante. Es la época en la cual la atención se dirige hacia los indicadores de rendimiento del personal académico (Bruneau y Savage, 2002; Morley, 2003), medido sobre todo por aspectos como el número de publicaciones o el presupuesto de los proyectos, la investigación y sus resultados representan un indicador para la promoción a los niveles profesionales superiores o para obtener «ventajas» profesionales. Este criterio evaluativo resulta discriminatorio para las mujeres. Los resultados obtenidos por Metz y Harzing (2012) sobre la poca presencia de mujeres en los consejos editoriales de las llamadas revistas de prestigio, es un indicador claro del sesgo existente. También el trabajo de Duch y otros (2012), sugiere que existe correlación entre el hecho de que las mujeres publiquen menos en revistas de impacto con el hecho de que obtienen menos recursos para investigar. Teniendo en cuenta que la investigación permite la orientación de la universidad hacia el mercado y hacia la sociedad (Olssen y Peters, 2005), conseguir un nombre en un campo de investigación, se convierte en una necesidad para poder avanzar en la carrera académica. 
Los criterios de calidad aplicados para la evaluación de la trayectoria profesional responden a la lógica de las ciencias «duras» y no a la de las ciencias «blandas» (Arranz, 2004). La evaluación de la productividad en investigación y los criterios empleados se consideran otro obstáculo en el desarrollo exitoso de la carrera de las mujeres. Se añaden las exigencias derivadas de la tensión entre la vida personal y la profesional (Forster, 2000; Probert, 2005), el tiempo dedicado a la maternidad y a la familia (Donoso, 2011; Mervis, 2012; Rafnsdóttir y Heijstra; Resick, 2012; Foa, 2012) o la falta de redes de trabajo colaborativo, lo que hace que el acceso a la financiación sea difícil para las investigadoras (Brink y Benschop, 2012).

A pesar de las evidencias, la mayoría de profesoras universitarias aún tiene poca conciencia sobre las desigualdades existentes y, en consecuencia, poca motivación para procurar cumplir con los criterios establecidos para promocionar (Arranz, 2004).

Aunque pocas, también existen profesoras que han alcanzado cierto grado de reconocimiento como investigadoras en sus áreas de conocimiento. Es especialmente interesante estudiar qué condiciones se han dado para que las barreras anteriormente citadas hayan sido superadas en sus casos. Cabe mencionar que hay menos investigación dedicada al estudio de los factores de éxito que a los obstáculos en la carrera académica de las profesoras. Los que hay han servido como base para nuestro estudio, abordan: los programas de formación y la importancia que tienen en la construcción profesional y en la adquisición de capacidades investigadoras para las profesoras (Devos, 2000; Ginther y Kahn, 2006) y los programas de «mentoring» en los que las profesoras participan (Groombdridge y Worden, 2003, Higgs, 2003 y Guillarmon, 2011) Las conclusiones derivadas de la convención de la «Association for Behavioral and Cognitive Therapies», celebrada en Nueva York en 2009, recogen experiencias de mujeres que han conseguido romper el llamado «techo de cristal» existente en la institución universitaria (McGinn y Newman, 2012).

Siguiendo estos trabajos anteriormente citados, nuestro estudio ha sido diseñado para investigar los factores específicos, las estrategias, las culturas de trabajo y los condicionantes asociados al éxito profesional de las mujeres investigadoras. En este planteamiento nos hemos guiado por las observaciones de Fox y Colatrella (2006, p. 377) que apuntan:

"Women's participation, performance and advancement are not a simple function of their individual characteristics, such as prestige of doctoral origins, training or skills. Rather their participation and attainments also reflect and are affected by features of organizational contexts in which they work including work climates and cultures, work structures, evaluative practices and reward patterns, among other factors." 
Este artículo ofrece un punto de partida para estudios posteriores que profundicen sobre los factores de éxito en las carreras académicas de profesoras universitarias y también puede servir como base para orientar las prácticas y las políticas en las instituciones de educación superior.

\section{METODOLOGÍA}

Hemos entrevistado a profesoras que lideran grupos de investigación consolidados en una universidad pública catalana. Todas las mujeres participantes son investigadoras reconocidas en su campo de conocimiento. Se ha adoptado el paradigma cualitativo, dado que el estudio se ha diseñado para proporcionar un acercamiento a las experiencias intelectuales y a la construcción de la carrera investigadora de las mujeres desde la percepción de las mismas protagonistas. Se pretende descubrir e interpretar sus vivencias personales. Se han entrevistado 7 académicas de diferentes campos disciplinares del área de las ciencias sociales. El grupo está formado por personas que tienen entre 30 y 60 años y son profesoras ayudantes doctoras (categoría denominada «lector/a» en Cataluña), titulares y catedráticas. La muestra elegida para este primer estudio exploratorio, aunque no es muy amplia, refleja la diversidad de experiencias y tipologías profesionales en las que se encuentran las profesoras con una trayectoria investigadora consolidada en general dentro de las Ciencias Sociales, el ámbito escogido como objeto de estudio en la investigación realizada.

Han servido de población de referencia los grupos de investigación reconocidos por la Generalitat de Catalunya en la convocatoria 2009-2013, denominados «Grups de Recerca de la Generalitat de Catalunya (SGR)». Se trata del máximo reconocimiento que los grupos de investigación de las universidades públicas y privadas de Cataluña pueden obtener y va asociado, frecuentemente, a cierta dotación económica destinada financiar proyectos de investigación del grupo.

La universidad estudiada cuenta con un número total de 69 grupos de investigación en ciencias sociales y jurídicas reconocidos por la Generalitat de Catalunya, de los cuales 45 son liderados por hombre y 24 por mujeres.

De estos 24 grupos liderados por mujeres: 6 son de Derecho; 1 de Pedagogía; 7 de diversas Didácticas, 2 de Antropología; 1 de Sociología; 2 de Psicología; 1 de Ciencias Económicas y 4 de Geografía.

De entre todos ellos, en una primera fase se seleccionaron 7 de las que accedieron a participar 4. En una segunda fase se amplió la muestra con 6 investigadoras más, 3 de las cuales pasaron a formar parte del estudio. Finalmente la muestra quedó compuesta por una investigadora de cada una 
de las siguientes disciplinas: Geografía, Antropología, Pedagogía, Sociología, Ciencias Económicas y dos investigadoras de distintas Didácticas. En el apartado dedicado a los resultados se reproducen citas textuales de las participantes en el estudio, por considerarse que ilustran la esencia de la categoría de contenido considerada. Se opta por no incluir ningún elemento identificativo a fin de preservar el anonimato, puesto que, como se decía más arriba, son todas profesoras de la misma universidad y son pocas, puesto que no hay muchas mujeres dirigiendo SGR en Ciencias Sociales.

Realizaron las entrevistas las mismas investigadoras durante el periodo comprendido entre diciembre del 2010 y febrero del 2011. Tuvieron una duración de entre 40 y 50 minutos. Se utilizó un guión de entrevista orientativo y abierto que se centraba en los siguientes tópicos de discusión:

- Los antecedentes y el contexto actual de la situación profesional de la investigadora.

- El papel de la investigación en relación a otras funciones propias de su perfil profesional.

- Los factores de éxito en la investigación según su propia percepción.

- Los factores organizativos y grupales.

- Los factores personales.

- Las dinámicas y culturas de trabajo.

- Otros tópicos.

Las entrevistas se transcribieron y fueron validadas por las mismas entrevistadas. Se realizó un análisis de contenido (Ruiz Olabuénaga, 2003) utilizando el programa de análisis de datos cualitativos MAXQDA 2007.

Se identificaron tres categorías de factores de éxito, cada una con una serie de subcategorías. En la tabla 1 se expone una síntesis de ellas:

\begin{tabular}{|l|l|}
\hline Categorías & Subcategorías \\
\hline $\begin{array}{l}\text { Condicionantes personales } \\
\text { del éxito }\end{array}$ & Vocación \\
& Pasión por el saber \\
& Dedicación al trabajo \\
& Sistema de acreditación y evaluación \\
& Conciliación de la vida profesional y la personal \\
\hline
\end{tabular}




\begin{tabular}{|l|l|}
\hline Categorías & Subcategorías \\
\hline $\begin{array}{l}\text { Condicionantes grupales } \\
\text { del éxito }\end{array}$ & $\begin{array}{l}\text { Estructura del grupo } \\
\text { Proceso de selección de los miembros } \\
\text { Estrategias de formación } \\
\text { Liderazgo ejercido en el grupo } \\
\text { Acciones de «mentoring» }\end{array}$ \\
\hline $\begin{array}{l}\text { Condicionantes institucionales } \\
\text { del éxito }\end{array}$ & $\begin{array}{l}\text { Relación docencia-investigación-gestión } \\
\text { Financiación } \\
\text { Gestión del tiempo } \\
\text { Soporte institucional } \\
\text { Autonomía }\end{array}$ \\
\hline $\begin{array}{l}\text { Ideas/recomendaciones para } \\
\text { que las investigadoras tengan } \\
\text { éxito }\end{array}$ & \\
\hline
\end{tabular}

Tabla 1. Categorías de análisis de las entrevistas y subcategorías surgidas

\section{RESULTADOS}

\section{Factores personales que contribuyen al éxito}

La construcción de la carrera investigadora empieza en el momento en que se finalizan los estudios doctorales o incluso antes, según nos confirma algunas de nuestra entrevistadas: "El tema de la investigación me ha gustado desde siempre». Esta etapa inicial en la cual la investigadora empieza a tener confianza en su capacidad, se caracteriza por «la perseverancia, la creatividad, la irregularidad, la ilusión y las ganas de mejora». Esta fase inicial es importante para entender las diferentes maneras de relacionarse con la investigación, el crecimiento y el desarrollo continuo desde ser una «aprendiz» hasta ser una investigadora de éxito (Akerlind, 2008).

En esta etapa de comienzo de la carrera investigadora, la formación postdoctoral, especialmente las estancias de investigación fuera del país, han sido mencionadas por todas las entrevistadas como factores determinantes: "Quizás hay una cosa importante, el haberme formado fuera y el dominar el inglés, esto para mí es fundamental, poder escribir los artículos directamente en inglés, poder publicar sistemáticamente fuera»; "abrir campos $y$ hacer contactos».

Ninguna de las entrevistadas ha desestimado el peso de la motivación, del trabajo duro, de la pasión por el campo de conocimiento, del interés temprano para la investigación y de la curiosidad por el saber. En este sentido nuestros resultados coinciden con estudios como el de Dever y Morri- 
son (2009) que apuntan hacia el papel fundamental de la motivación por encima de cualquier aspecto institucional o grupal en la construcción de las carreras investigadoras.

Una serie de características individuales completan el cuadro de condicionantes personales para el éxito investigador: la paciencia, el control de la ansiedad, la constancia en el trabajo "porque cuando investigas al inicio ves una lucecita a lo lejos y cuesta mucho»; "[es fundamental] ser muy perseverante, muy tenaz, muy segura de lo que tú quieres hacer, pero también muy abierta a mejorar».

A estos elementos se añaden aspectos que tienen que ver con el rol de la familia, con el apoyo que se recibe en los contextos externos a la universidad en los comienzos de la carrera académica. La mayoría de las expertas participantes proceden de familias con un nivel cultural alto, que las que han apoyado en su decisión de dedicarse a estudiar: "considero que un aspecto clave en mi trayectoria profesional ha sido mi familia. Mi padre es psiquiatra y profesor universitario...» El factor «procedencia social» ha sido clave para que algunas mujeres en la historia de la humanidad hayan podido dedicarse a la ciencia (Sánchez Prieto, 2010) y por lo que parece actualmente sigue siéndolo.

Una vez iniciada la carrera académica, se han de pasar una serie de evaluaciones externas sobre los propios méritos. Las llamadas acreditaciones se han convertido en una medida del éxito investigador y en un condicionante para poder seguir el desarrollo profesional en sus inicios. Las valoraciones de dichos procesos de acreditación por parte de las investigadoras participantes son desiguales. Aquellas que ya están consolidadas tienden a pensar que el nuevo modelo es un elemento motivador, que traza una dirección a seguir en la carrera profesional: "Yo digo que los jóvenes han entrado con estas reglas del juego, yo creo que sí que la gente se implicará mucho más en la investigación y no solo en ella, sino también en la difusión de los resultados de investigación. Nos ayudará a parecernos más a las titulaciones de ciencias». Las que están en vías de consolidación, por otra parte, comentan las dificultades debidas a la gestión de los tiempos: "Hay momentos vitales en que se hace imposible responder a ellos [a los sistemas de acreditación], tiene que revisarse como se reequilibran las dedicaciones y las tareas, si no, no funciona. Por tanto, que toda la evaluación del profesorado pivote en un porcentaje tan alto sobre los resultados de la investigación es terriblemente injusto».

Muchas de las entrevistadas muestran inquietud porque se sobrevaloran algunos aspectos de la producción científica, especialmente los artículos de impacto. En cambio, a otros productos como los informes o los 
libros no se les da mucha importancia en el sistema actual de evaluación: "que un artículo vaya a parar a una revista, yo sé que es importante, ipero que no se pueda valorar nada más que eso![…] yo he realizado informes para la comisión europea que nunca se han publicado, ni se publicaran nunca, que son verdaderas (iba a decir joyas) cosas muy interesantes y muy bien hechas... Bien, estos informes al no estar publicados en una revista con impacto, pues bien: ifuera!

¿Cómo se enfrentan las mujeres a estos retos profesionales y construyen una carrera exitosa? Como ya se ha visto, trabajando duro, mostrando pasión por la investigación y siendo competitivas. A esto se le añade la importancia de «los modelos», «la identificación con los mentores» y «la acción colectiva». Nuestros resultados coinciden con los de Bagilhole (1994) que identifica diferentes estrategias que las mujeres utilizan para superar los obstáculos en la carrera investigadora, entre los cuales: la dedicación al trabajo y el apoyarse en las redes profesionales. En nuestro caso, las participantes reconocen que dedican al trabajo mucho esfuerzo y que invierten mucho tiempo: «siempre de vacaciones con la maleta llena de libros, no siempre encerrada en casa, también tenía la suerte de trabajar en otros sitios, cerca del mar, pero el trabajo siempre ha sido una constante»; "[para investigar] es necesaria una cierta continuidad diaria, no ocho horas diarias, que es muy difícil, pero sí un tiempo todos los días»., "despertarse temprano por la mañana» para poder tener la "tranquilidad para escribir» $\mathrm{o}$ "negociar las tareas domesticas», son otras de las referencias que las participantes hacen en relación al tiempo dedicado al trabajo.

A estos elementos se les añaden otros factores que favorecen el éxito, especialmente los que tienen que ver con tener modelos y con el mentoring. Las participantes describen a sus mentores/as como las personas que les han marcado el camino, dándoles las pautas necesarias y ofreciéndoles apoyo en los momentos difíciles: "Es muy importante, tener una pauta institucionalizada y una persona que es clave cuando tu eres joven para decirte: el camino es este, porque éste es el que yo he seguido, éste es el que me ha ido bien y éste es el que la academia reconoce, y por tanto el camino este». Nuestras entrevistadas señalan que la universidad es un lugar difícil para sobrevivir sin apoyo, en consonancia con los resultados de Caplan (1993) y de Toth, (1997), entre otros. En este sentido una de las participantes añade, refiriéndose al papel del mentor: «debe haber una persona que sea capaz de decir: hay que ir por aquí, lo tenemos claro, vamos a ir por aquí, nos vamos a encontrar dificultades como nos pasa a lo largo de la vida, pero las vamos a superar, tú ves haciendo que yo te doy una mano para que tu vayas continuando».

Así, el mentor y la tarea que lleva a cabo son aspectos clave y tienen un papel importante (Acker y Armenti, 2004) en la construcción de una 
carrera exitosa. El poder de las redes y del grupo ha sido ampliamente debatido por autores como Davies (2003) o McLaren (2002) y nuestro estudio confirma sus resultados. "El grupo añade otra dimensión, el trabajar con mucha gente que te hacen ver desde perspectivas distintas, cosas que no se te habian ocurrido […] son siempre un elemento de avance y de placer intelectual extraordinario».

Esto nos lleva a otro aspecto importante que contribuye al éxito profesional: el gusto por compartir, la cultura de la colaboración: «la red de ayuda siempre beneficia al grupo y si beneficia al grupo, beneficia a los individuos».

\section{Factores grupales que contribuyen al éxito}

El grupo de investigación puede convertirse en una de las estrategias más importantes para asegurar el éxito en la actividad investigadora. Las entrevistadas se sitúan en relación al tema entre dos polos: por un lado consideran la autonomía en el trabajo como un aspecto clave y muy importante para el éxito, asociado con la fase inicial de su trayectoria investigadora y por otro lado, destacan las potencialidades de la actividad en grupo, en fases posteriores, una vez probada su valía investigadora individual.

La autonomía se relaciona con la independencia en el trabajo: «Yo por lo menos, el entorno que tengo aquí de trabajo me permite hacer lo que quiera», "al principio yo trabajaba sola», "trabajo en casa por la mañana», aspectos debatidos en otros estudios (Lambright y Teich 1981). Se configura de esta manera la imagen del investigador como un científico libre y autónomo, a veces aislado (Travaille y Hendriks, 2010) y se considera que debe buscarse el equilibrio entre la internalización y la socialización en la producción del conocimiento puesto que los resultados demuestran la importancia de la socialización en una trayectoria investigadora de éxito. Así mismo, las participantes explican que tanto el proceso de redacción de artículos, el de ejecución de proyectos o la participación a las actividades formativas se hacen en grupo. Muchas apuntan entre los factores que están implicados en la creación de conocimiento, la colaboración con los demás miembros del grupo, "las reuniones de trabajo», "las interacciones con los especialistas» y la "participación en seminarios de intercambio». Así estos resultados concuerdan con los obtenidos por Grbich (1998).

Todos los grupos de las personas entrevistadas obtienen fondos económicos «modestos» para la investigación. Las investigadoras están convencidas de que un elemento importante para que sus proyectos ganen concursos es que solicitan poco dinero, además de saber avanzarse a las necesidades sociales en relación a la investigación: "tener olfato para el tema, es decir, 
haber sabido con tiempo qué temas serian interesantes de estudiar, ser pionero en un tema es fundamental».

La obtención de recursos compitiendo con otros grupos de investigación es un hecho que llena de orgullo a los miembros del equipo y, por tanto, además de ser un necesario el dinero para poder investigar, supone también reconocimiento y aumenta la moral del grupo: "hace dos años nos dieron un Exploratory Workshop, y casi estoy más orgullosa de eso que del SGR, ese es el resultado más visible del trabajo de investigación». Este resultado coincide con una de las conclusiones a la que llegan McLaughlin y Helsi (2013) en su estudio: a pesar de lo que comúnmente se cree, las mujeres hacen más solicitudes para recibir fondos para la investigación que los hombres.

También coinciden las participantes es destacar la importancia que dan a la formación dentro de sus grupos de trabajo: : «una de las cosas que hacemos es invitar algún experto o experta que está trabando en lo nuestro»; "una vez al mes nos reunimos todo el equipo y hacemos dos tareas: nos ponemos al día sobre cómo van los proyectos y demás y después hacemos alguna actividad formativa»; "la formación es fundamental para el desarrollo de los investigadores jóvenes "trabajar en equipo es clave para el éxito. Saber llevar un equipo que tenga buen clima, en el que haya cooperación, es fundamental. De hecho yo delego mucho. Hay investigadores que están suficientemente formados para dirigir sus propios proyectos», igual que la «generosidad» de los investigadores sénior en compartir el conocimiento: "porque mis conocimientos, que son un pequeño grano de arena en el proceso de avanzar la ciencia, quiero que esos conocimientos vayan a más gente, se difundan y lleguen a más gente».

Sobre el estilo de liderazgo ejercido, las participantes difieren poco. Parece que valoran el poder delegar responsabilidades y, cuando perciben que pueden, lo hacen. Otro aspecto común es que consideran que su tarea fundamental consiste en apoyar a las personas para que puedan desarrollar sus carreras profesionales individuales dentro del grupo, respetando los ritmos de trabajo individuales. Para ello ocasionalmente han de ejercer de mediadoras en los "pequeños» conflictos de intereses que pueden darse entre los miembros. También todas ellas consideran fundamental de su papel como líderes el velar para que el clima del grupo sea positivo, que ir a trabajar sea algo agradable.

Ninguna de las investigadoras se ha formado específicamente para el ejercicio del liderazgo aunque todas ellas mencionan las bondades que en su opinión tendría el hecho de participar en actividades de formación relacionadas con el tema: «no, no he hecho ningún tipo de formación específica para 
liderar, pero quizá debería de hacerla puesto que ahora en mis «vejeces» lo que más hago es liderar...»; "funciona un poco así intuitivamente...».

\section{Factores institucionales que contribuyen al éxito}

Aparte de los factores personales y de los grupales, los institucionales también influyen sobre el éxito profesional de quien investiga. El papel de los departamentos y de las facultades es decisivo.

El hecho de que haya otros grupos de investigación en la organización, en algunos casos se vive como un estímulo para la mejora continua. Una de las participantes, además, añade un concepto interesante en este punto: las redes de apoyo horizontales que puedan establecerse entre mujeres pertenecientes a diferentes núcleos dentro de la misma organización y hasta pertenecientes a otras organizaciones: "creo que especialmente para las mujeres es muy importe construirse redes horizontales dentro de los ámbitos académicos, tener no tanto el mecanismo vertical del mentor que te ayuda desde arriba, si no […] el mecanismo horizontal de redes de mujeres que están haciendo lo mismo que nosotras en otros sitios, en otros departamentos, en otras universidades, dentro y fuera de España que pueden trabajar en lo mismo que nosotras o simplemente podemos tener los mismos problemas».

Otras participantes, sin embargo, afirman que no perciben influencia directa de la organización en el desarrollo de sus equipos de trabajo. Una de ellas afirma que las exigencias organizacionales condicionan negativamente el éxito de su equipo en tanto que consumen tiempo que debería poder destinarse a la investigación: "yo no tengo la sensación, y quizá sea injusta, de que la Universidad me haya dado apoyo con la investigación, no la tengo. Las ayudas económicas que he recibido han sido siempre muy pequeñas. El dinero que he conseguido ha sido de la Unión Europea».

La asignación de la docencia se realiza en las facultades y la relación entre docencia e investigación puede constituir un factor de éxito profesional. Los estudios que han analizado la relación entre la docencia y la productividad en investigación no indican una correlación directa (Hattie y Marsh, 1996). Sin embargo, autores como Griffiths (2004) insisten sobre el carácter complejo de las dos actividades que requieren de mucha dedicación. Nuestras participantes corroboran este resultado: «la docencia siempre quita mucho tiempo».

Si tenemos en cuenta que a las mujeres se las asocia más a la docencia que a la investigación (Poole, Bornholt y Summers, 1997), es una clara ventaja que los contenidos docentes coincidan con los de investigación: «lo 
más óptimo es que la docencia pueda estar relacionada con aquello que investigas de manera que puedas explicar en clase aquello que has investigado». El problema es que muchas veces la coincidencia no se da y se vive como un problema: "cuando esté en una posición consolidada, si lo consigo que espero que sí, podré escoger y para yo creo que es muy importante la vinculación entre docencia e investigación».

Por otro lado las entrevistadas identifican los beneficios que la dirección de trabajos de investigación y supervisión de tesis de doctorado tienen para su propia carrera investigadora: «la docencia en Máster y Doctorado, y tener alumnos y dirigir tesis doctorales, eso sí te compensa de muchas cosas», coincidiendo con las conclusiones de Dever y Morrison (2009).

En el caso de las actividades de gestión, las académicas consideran que «la gestión es brutal, nos toca hacer unas cosas alucinantes y tomar decisiones y saber de todo, contabilidad para hacer presupuestos y no sé cuantas cosas más» y es una responsabilidad que "quita mucho tiempo». Este resultado está en la misma línea que el obtenido por McLaughlin y Helsi (2013), mencionado en la introducción, según el cual a las mujeres se les pide que ejerzan cargos de gestión en mayor número que a los hombres y ellas tienden a acceder. Pero se trata de cargos que conllevan mucho trabajo y poco poder para tomar decisiones importantes.

El hecho de ser mujer ha sido más un obstáculo que una ventaja para construir una carrera académica brillante. Y lo sigue siendo si partimos de los estudios realizados en España (Guillamon, 2011), en otros países europeos (Seierstad y Healy, 2012; Macarie y Moldovan, 2012), en países norteamericanos (Duch y otros, 2012) y de las afirmaciones que hacen las profesoras entrevistadas en esta investigación: «es un campo en que a pesar de que hay muchas estudiantes, muchas doctoradas y muchas mujeres iniciando la carrera, tenemos sólo 4 catedráticas en toda España y están desesperadas con las oposiciones de cátedra porque tienen que estar recorriéndose toda España para los tribunales, estoy en un campo que no puedo decir que sea especialmente "friendly» para las mujeres».

Trabajos como los de Brink y Benschop (2012) ponen claramente en duda que «meritocracia» sea una vía objetiva para garantizar el acceso a la carrera académica y la promoción una vez en ella. Cuando las académicas toman conciencia sobre la desigualdad de género aun vigente, se movilizan en el sentido de que actúan para intentar la erradicación de la desigualdad: "son muy pocas [las mujeres que están], yo por ejemplo he estado dirigiendo una revista y he sido la única mujer del comité editorial durante muchos años, hasta que yo me he ido y he podido meter a otras dos [mujeres]». 


\section{CONCLUSIONES}

El concepto de «investigación de éxito» comprende la calidad y la cantidad de las producciones científicas, la reputación de quien investiga y su prestigio en el campo. Connota el reconocimiento de que se ha contribuido al desarrollo del campo de conocimiento y al desarrollo profesional y personal de los compañeros y supone una contribución social relevante.

El éxito también se relaciona con la organización en la que se trabaja y depende en gran medida del funcionamiento del grupo. El grupo es un factor de éxito si se procura el desarrollo individual de cada miembro. Liderar para compartir y acompañar es primordial para el buen rendimiento grupal. Un grupo es exitoso cuando tiene suficientes resultados (publicaciones, proyectos financiados conseguidos, formación), un buen clima de trabajo asociado con unas condiciones de infraestructura de financiación adecuadas y una buena reputación de la investigadora líder. El equipo es un motor necesario para llegar al éxito porqué sus resultados son más que la suma de los resultados individuales y dicha sinergia es imprescindible cuando la carrera académica cada vez es más competitiva.

Como cualidades personales para la investigación destacan: la pasión por la ciencia, combinada con la perseverancia, la curiosidad y la iniciativa, en un contexto en el cual se tiene suficiente autonomía.

A lo largo de este artículo hemos analizado la percepción sobre género e investigación de investigadoras que han conseguido tener un lugar destacado entre la comunidad científica, comprometidas con la producción de resultados de investigación y con el crecimiento de sus ámbitos de conocimiento, a pesar de no tener en muchas ocasiones, la infraestructura y el tiempo suficientes para ello. Son académicas que enfrentándose a las dificultades de formar parte de una cultura organizativa masculinizada, han decidido seguir trabajando y creer en la posibilidad de cambiar esta realidad. 


\section{REFERENCIAS BIBLIOGRÁFICAS}

Acker, S. y Armenti, C. (2004). Sleepless in academia. Gender and Education, $16,13-24$.

Akerlind, G.A. (2008). Growing and developing as a university researcher. Higher Education, 55, 241-254.

Amâncio, L. (2005). Reflections on science as a gendered Endeavour: changes and continuities. Social Science Information, 44 (1), 65-83.

Arranz L.F. (2004). Las mujeres y la universidad española: estructuras de dominación y disposiciones feminizadas en el profesorado universitario. Política y Sociedad. 41 (2), 223-242.

Asmar, C. (1999). Is there a gendered agenda in academia? The research experience of female and male $\mathrm{PhD}$ graduates in Australian universities. Higher Education, 38 (3), 255-273.

Bagilhole, B. (1994). Being different is a very difficult row to hoe: survival strategies of women academics. En S. Davies; C. Lubelska y J. Quinn (eds.) Changing the subject: Women in higher education. (pp. 211-231). London: Taylor \& Francis.

Bagilhole, B. (2007). Challenging women in the male academy: think about draining the swamp. En P. Cotterill, S. Jackson y G. Letherby (eds) Chanllenges and negotiations for women in higher education. (pp. 21-32). Dordrecht: Springer.

Bagilhole, B. y White, K. (2003). Created in their image: An analysis of male cultural hegemony in higher education in Australian and the United Kingdom. En B. Groombridge y V. Mackie (eds.) Re-searching research agendas: women, research and publication in higher education. Proceedings of the Australian
Techology network-women's executive development (ATN-WEXDEV) 2003 Research Conference (1-12). Perth: Curtin University of Technology Learning Support Network.

Bermúdez, M.P.; Guillén-Riquelme, A.; Gómez-García, A.; Quevedo-Blasco, R.; Sierra, J.C. y Buela-Casal, G. (2011). Análisis del rendimiento en el doctorado en función del sexo. Educación XX1, 14 (1), 17-33.

Brink, M. y Benschop, Y. (2011). Salaying the Seven-Headed Dragon: The Quest for Gender Change in Anademia. Gender, Work and Organitzation, 19 (1), 71-92.

Brink, M. y Benschop, Y. (2012). Gender Practices in the construction of academic excellence: Sheep with five legs. Organitzation, 19 (4), 507-524.

Britton, C. (1999). Supporting women in research. En S. Hatt, J. Kent y C. Britton (eds.) Women, research and careers. (pp. 69-88). Houndsmills: Macmillan.

Bruneau, W.A., y Savage, D.C. (2002). Counting out the scholars: How performance indicators undermine universities and colleges (A CAUT Series Title) Toronto: James Lorimer.

Caplan, P. (1993). Lifting a ton of feathers: a woman's guide to surviving in the academic world. Toronto: University of Toronto Press.

Davis, B. (2003). Death to critique and dissent? The policies and practices of new managerialism and of «evidencebased practice», Gender and Education, 15 (1), 91-103.

Dean, E., Jonhson, L., Jones, G., y Lengkeek, N. (1996). Women, research and research productivity in the post-1987 universities: Opportunities and con- 
straints. University of Western Sydney, Nepean: Department of Employment, Education, Training and Youth Affairs, Evaluations and Investigations program, Higher education Division.

Dever, M. y Morrison, Z. (2009). Women, Research Performance and work Context. Tertiary Education and Management, 15 (1), 49-62.

Devos, A. (2000). Women Research21: Responding to the issue for beginning women academics. HERDSA News, 22 (2), 11-13.

Doherty, L., y Manfredi, S. (2005). Improving women's representation in senior positions in the higher education sector, stage findings. Oxford: Centre for Diversity Policy Research, Oxford Brookes University.

Donoso, T.; Figuera, P. y RodríguezMoreno, M.L. (2011). Barreras de género en el desarrollo profesional de la mujer universitaria. Revista de Educación, 355, 187-212.

Duch, J.; Zeng, X.; Sales-Pedro, M.; Radicchi, F.; Otis, S.; Woodruff, T.; y Amaral, L. (2012). The possible role of resource requirements and academia career-choice risk on gender differences in publication ate and impact. Plose One, 7 (12), e51332.

Flecha, C. (1999). Género y Ciencia. A propósito de los «estudios de la mujer» en las universidades. Educación XX1, 2, 224-244.

Foa, E.B. (2012). How being female influenced my professional experiences and growth. Behavior Therapy, 43, 715-717.

Forster, N. (2000). A case study of women academics"views on equal opportunities, career prospects and work-family conflicts in a British univesrity. Women in management review, 15 (7), 316-330.
Fox, M.F. y Colatrella, C. (2006). Participation, performance and advancement of women in academic science and engineering. What is the issue and why. Journal of Technology Transfer, 31(3), 377-386.

Ginther, D. y Kahn, S. (2006). Women's Careers in Academic Social Science: Progress, Pitfalls, and Plateaus.

Ginther, D. (2003). Is MIT an Exception? Gender Pay Differences in Academic Science. Bulletin of Science Technology Society, 23, 21-26.

Godden, J. (1996). From new to successful researcher: Enhancing the research skills of academics. En J. Godden, A. Brew, y E. Roe (eds.) Learning research skills: Programs and issue: reports on the research skills development program for academic staff 1990-1995. (pp. 4-15). Sydney: University of Sydney Centre for Teaching and Learning.

Grbich, C. (1998). The academic researcher: Socialization in settings previously dominated by teaching. Higher Education, 36, 67-85.

Griffiths, R. (2004). Knowledge production and the research-teaching nexus in eight advanced-industrialized countries. Higher Education, 34 (3), 397420.

Groombridge, B., y Worden, S. (2003). Values, visions, strategies and goals: Is coaching a viable pathway? En $\mathrm{B}$. Groombridge, y V. Mackie (eds.) Researching research agendas: Women, research and publication in higher education: Proceedings of the Australian Technology Network-Women's Executive Development (ATN-WEXDEV) 2003 Research Conference. (pp. 117185). Perth: Learning Support Network, Curtin University of Technology.

Guillamon, C. (2011). Los condicionantes de la carrera investigadora en la 
Universidad que encuentran las mujeres. En M. Tomàs (coord.) La Universidad vista desde una perspectiva de género. (pp. 77-112). Barcelona: Octaedro.

Hattie, J. y Marsh, H.W.(1996). The relationship between research and teaching: A meta-analysis. Review of Higher Education, 66, 507-542.

Higgs, J. (2003). Making a difference. En H. Edwards, D. Baume, y G. Webb (eds.), Staff and educational development: Case studies, experience and practice from higher education. (pp. 29-36). Sterling, VA/London: Kogan Page.

Hobson, J., Jones, G., y Deane, E. (2005). The research assistant: Silenced partner in Australia's knlowlegde production? Journal of Higher Education Policy and Mangement, 27 (3), 357-366.

Lambright, W.H., y Teich, A.H. (1981). The organizational context of scientific research. En P.C. Nystrom y W.H. Starbuck (eds.), Handbook of organizational design. (pp. 305-319). Oxford: Oxford University Press.

Macarie, F.C. y Moldovan, O. (2012). Are universities role models for communities? A gender perspective, Transylvanian Review of Administrative Sciences, 81-97.

McGinn, L.K. y Newman, M.G. (2012). Overcoming the glass ceiling. Lessons learned in looking back: Perspectives from trailblazers. Behavior Therapy, 43, 698-700.

McLaren, M. (2002). Feminism, Foucault and embodied subjectivity. Albany: State University of New York Press.

McLaughlin, S. y Helsi, V.L. (2013). Women don't ask? Women don't say no? Bargaining and service in the political science profession. The Profes- sion, April, 355-369.

Mervis, J. (2012). Is Motherhood the biggest reason for academia's gender imbalance? Science, 335, 1030-1031.

Metz, I. y Harzing, A. (2012). An update of gender diversity in editorial boards: a longitudinal study of management journals. Personnel Review, 41 (3), 283-300.

Milkman, K.L.; Akinola, M. y Chugh, D. (2012). Temporal distance and discrimination: an audit study in academia. Psychological Science, XX (X), 1-8.

Morley, L. y Walsh, V. (eds.). (1995). Feminist academics: creative agents for change. London: Taylor \& Francis.

Morley, L. (2003). Quality and Power in higher education. Buckingham: Open University Press.

Olssen, M. y Peters, M. (2005). Neoloberalism, higher education and the knowledge economy. From the free market to knowledge capitalism. Journal of Education Policy, 20 (3), 313-345.

Poole, M., Bornholt, L. y Summers, F. (1997). An international study of the gendered nature of academic work: Some cross-cultural explorations. Higher Education, 34 (3), 373-396.

Poole, M., Bornholt, L. y Summers, F. (1997). An international study of the gendered nature of academic work: Some cross-cultural explorations. Higher Education, 34 (3), 373-396.

Probert, B. (2005). I just couldn't fit it in: Gender and unequal outcomes in academic careers. Gender, Work and Organization, 12(1), 50-72.

Rafnsdóttir, G.L. y Heijstra, T.M. (2013). Balancing work-family life in academia: The power of time. Gender, 
Work and Organization, 20 (3), 283-296.

Ramsden, P. (1999). Predicting instructional research performance from published indicators: A test of a classification of Australian university types. Higher Education, 37 (4), 341-358.

Resick, P.A. (2012). Getting out of our own way. Behavior Therapy, 43, 708-711.

Ruiz Olabuénaga, J.I. (2003). Metodología de la investigación cualitativa. Bilbao: Universidad de Deusto.

Sagaria, M. y Agans, L. (2006). Gender equality in US higher education: International framing and institutional realities. Higashi-Hiroshima City: research Institute for Higher Education, Hiroshima University.

Sánchez Prieto, A.B. (2010). La educación de la mujer antes del año 1000. ¿Es Duhoda un caso único? Educación XX1, 13 (2), 69-94.
Seierstad, C. y Healy, G. (2012). Women's equality in the Scandinavian academy: a distant dream? Work, Employment and Society, 26 (2), 296-313.

Smeby, S. y Try, S. (2005). Departmental contexts and faculty research activity in Norway. Research in Higher Education, 46 (6), 593-619.

Tomàs, M., Duran, M.M., Guillamon, C. y Lavié, J.M. (2008). Profesoras universitarias y cargos de gestión. Contextos educativos, 11, 113-129.

Toth, E. (1997). Ms Mentor's impeccable advice for women in academia. Philadelphia: University of Pennsylvania Press.

Travaille E., y Hendriks, P. (2010). What keeps science spiralling? Unravelling the critical success factors of knowledge creation in university research. Higher Education, 59, 423-439. 


\section{PALABRAS CLAVE}

Género, Universidad, Carrera académica, Investigación.

\section{KEYWORDS}

Gender issues, University, Academic career, Research.

\section{PERFIL ACADÉMICO Y PROFESIONAL DE LAS AUTORAS}

Mar Duran-Bellonch, licenciada en Filosofía y Letras y doctora en Pedagogía. Es profesora del Departamento de Pedagogía Aplicada de la Universidad Autónoma de Barcelona y está especializada en el ámbito de la Dinámica de Grupos en organizaciones educativas. Además, investiga sobre género y carrera académica y sobre la evaluación de la formación en la empresa.

Georgeta Ion, licenciada en Psicología y Ciencias de la Educación y doctora en Ciencias de la Educación. Es profesora del Departamento de Pedagogía Aplicada de la Universidad Autónoma de Barcelona. Sus líneas de investigación se relacionan con la evaluación basada en competencias y el estudio de las instituciones de educación superior.

Dirección de las autoras: Departamento de Pedagogía Aplicada, G6, 255 y 248

Facultad de Ciencias de la Educación

Campus de Bellaterra 08913 - Cerdanyola del Vallès.

E-mail: mariadelmar.duran@uab.cat georgeta.ion@uab.cat

Fecha Recepción del Artículo: 01. Noviembre. 2011

Fecha Revisión del Artículo: 27. Febrero. 2012

Fecha Aceptación del Artículo: 27. Marzo. 2012

Fecha de Revisión para publicación: 24. Junio. 2013 
